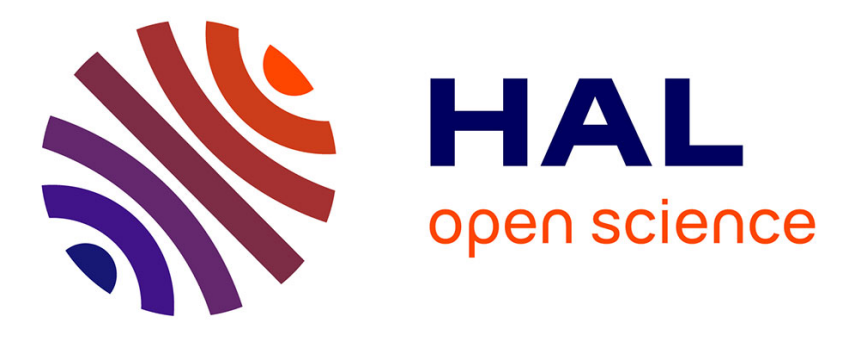

\title{
MAC protocol for volcano monitoring using a wireless sensor network
}

Loïc Le Guennec, Nancy El Rachkidy, Alexandre Guitton, Michel Misson, Karim Kelfoun

\section{- To cite this version:}

Loïc Le Guennec, Nancy El Rachkidy, Alexandre Guitton, Michel Misson, Karim Kelfoun. MAC protocol for volcano monitoring using a wireless sensor network. Network of the Future (NoF), 2015, Montreal, Canada. pp.1 - 5, 10.1109/NOF.2015.7333310 . hal-01471667

\section{HAL Id: hal-01471667 \\ https://hal.uca.fr/hal-01471667}

Submitted on 20 Feb 2017

HAL is a multi-disciplinary open access archive for the deposit and dissemination of scientific research documents, whether they are published or not. The documents may come from teaching and research institutions in France or abroad, or from public or private research centers.
L'archive ouverte pluridisciplinaire HAL, est destinée au dépôt et à la diffusion de documents scientifiques de niveau recherche, publiés ou non, émanant des établissements d'enseignement et de recherche français ou étrangers, des laboratoires publics ou privés. 


\title{
MAC Protocol for Volcano Monitoring using a Wireless Sensor Network
}

\author{
Loïc Le Guennec ${ }^{(1,2)}$, Nancy El Rachkidy ${ }^{(1,2)}$, Alexandre Guitton ${ }^{(1,2)}$, Michel Misson ${ }^{(3,2)}$, Karim Kelfoun ${ }^{(1,4)}$ \\ (1) Clermont Université, Université Blaise Pascal, BP 10448, F-63000 Clermont-Ferrand, France \\ (2) CNRS, UMR 6158, LIMOS, F-63175 Aubière, France \\ (3) Clermont Université, Université d'Auvergne, LIMOS, BP 10448, F-63000 Clermont-Ferrand, France \\ (4) Centre de Recherches Volcanologiques and CNRS, UMR 6524, Magmas et Volcans, 63038 Clermont-Ferrand \\ Cedex, France \\ Emails: \{leguennec,nancy,guitton,misson\}@ sancy.univ-bpclermont.fr, k.kelfoun@opgc.univ-bpclermont.fr
}

\begin{abstract}
Many existing MAC protocols for wireless sensor networks try to achieve simultaneously low latency and low power consumption. For many monitoring applications, however, there are two separated types of traffic. Most of the traffic is periodic, and is not subject to latency constraints: energy savings is the main objective for this traffic. A small proportion of the traffic has strict latency requirements, and the energy consumed for this priority traffic is not an issue. In this paper, we introduce a MAC protocol that can achieve this traffic differentiation in a WSN. We propose to combine the low power listening approach for periodic traffic with opportunistic encounters mechanisms for urgent traffic. We show that our approach provides a good ratio of consumed energy per delivered packet of the periodic traffic, while keeping a low loss rate for the priority traffic.
\end{abstract}

\section{INTRODUCTION}

Wireless Sensor Networks (WSNs) cover a wide range of applications, including the monitoring of natural sites [1], [2]. Volcanoes are natural sites that are typically difficult to monitor, due to dangerous access. Deploying disposable wireless sensor nodes for extended periods is a way to collect environmental data periodically, without risking the loss of costly hardware when the volcano erupts. Two types of traffic can be identified for volcano monitoring: periodic traffic and urgent traffic. (i) Periodic traffic is collected when the volcano is sleeping, and the data collection has to be energyefficient so that the WSN can last for several months without human intervention. Periodic traffic is usually archived in large databases and processed to build long-term models. Thus, they have loose latency requirements. (ii) Urgent traffic is produced when the volcano becomes active. In this case, the traffic has to be forwarded quickly for two reasons: nodes might be destroyed during seismic events, and the data obtained during volcanic activity is important for scientists. Energy-efficiency is not an issue when these rare events occur.

There are many MAC protocols for WSNs that combine both energy constraints with latency constraints. They often achieve a trade-off of these two objectives. They are usually based on duty-cycling: each node alternates between a sleep state where nodes save energy, and a wake state where nodes can communicate. The BMAC [3] protocol introduced a low power listening approach based on preambles in order to save energy at the receiver side. In this protocol, sender nodes transmit a long preamble before transmitting the actual data. Receivers wake up periodically to assess the channel. When they detect a preamble, they wait for the data. Otherwise, they go back to sleep. This approach has been improved in the literature in order to reduce the reception time of receivers.

However, our volcano monitoring application suggests two types of traffic that are independent (note that they are not generated at the same time, for instance). Most of the traffic consists of periodic data, which are not subject to latency requirements. However, the urgent traffic has to be delivered as quickly as possible, generally in a bounded time in order to be of value for the scientists. Thus, the main goal is not to have a MAC protocol that achieves a good trade-off in energy consumption and in latency, but a protocol that is able to deal with QoS differentiation at the MAC layer.

In this paper, we introduce a new MAC protocol for volcano monitoring with a WSN. Our protocol achieves QoS differentiation at the MAC level. Urgent traffic is sent using an asynchronous sender-initiated approach based on the XMAC protocol. Periodic traffic is sent using an opportunistic mechanism that takes advantage of the duty-cycle mechanism. In addition, our protocol is able to exploit the convergence of data to a single destination, called sink: each node can transmit its data to the first neighbor towards the sink which wakes up rather than having to wait for a specific neighbor to wake up, thus dramatically reducing latency.

The rest of this paper is organized as follows. Section II presents a brief state of the art of MAC protocols for WSNs. Section III presents a description of our protocol. Section IV discusses the simulation results. Section V presents an overview of the prospects and concludes this paper.

\section{RELATED WORK}

Energy consumption is a critical issue in most WSN applications [4]. Duty-cycling the radio is considered the most relevant technique to achieve power efficiency in the MAC sublayer [5], as the radio module consumes the largest amount of energy among all the node components. Duty-cycling the radio allows nodes to switch periodically between an active state and an inactive state.

Synchronous MAC protocols alternate active and inactive periods, such that all nodes share their active periods at 
the same time [6]. This is achieved by implementing a synchronization mechanism at the beginning of the active period. Example of synchronous MAC protocols include SMAC [7], DW-MAC [8], or the beacon-enabled mode of IEEE 802.15.4 [9]. The main advantage of synchronous MAC protocols is that all nodes share a common active period, with a limited period of idle listening. The main disadvantages are the following: the synchronization is generally complex and introduces a significant overhead, and the contention for the medium is high during the active period.

Asynchronous MAC protocols are based on independent schedules of active and inactive periods: the active period of a node might not match the active period of another node. Asynchronous MAC protocols have many advantages: they do not require synchronization, they are conceptually distributed, they are tolerant to network dynamicity, and nodes can achieve lower duty-cycles [10].

Asynchronous MAC protocols can be classified into senderinitiated protocols and receiver-initiated protocols. In receiverinitiated MAC protocols, receivers trigger communications by transmitting a probe. When active, the sender remains silent even if it has data to transmit, until it receives the probe from the receiver. Upon receiving this probe, the sender starts the transmission. The main advantage of receiver-initiated protocols is that the length of the probe is shorter than the preamble of sender-initiated protocols, which decreases transmission latency and channel occupancy. The drawbacks of receiver-initiated protocols are the following: (i) the cost of the communication is spent by the receivers, which are more numerous than senders in a WSN, (ii) frequent probe transmissions can cause channel congestion and delay data communications, which negatively impact the scalability.

In sender-initiated MAC protocols, the sender triggers the communications. A preamble is used to notify receivers about upcoming data transmission. The length of the preamble is set to a duration that is longer than the sleep period of the receiver, in order to ensure that the receiver wakes up during the preamble. The preamble also prohibits other neighboring nodes from transmitting, which reduces collisions. B-MAC [3] sends a preamble with fixed length before starting data transmission. Once the preamble is detected, the node remains active until the end of the transmission or until the node switches to sleep mode. WiseMAC [11] allows receivers to piggyback their next wake up time in acknowledgment frames, which reduces the length of further preambles. X-MAC [12] uses short preambles to reduce the length of preambles.

In this paper, we focus our comparison on the X-MAC protocol, which is an asynchronous, sender-initiated MAC protocol. X-MAC [12] decreases the length of preamble by transmitting short preambles containing the destination address. When a receiver wakes up, it waits for the end of a short preamble, decodes the destination address, and can decide whether to wait for the frame (at the end of the sequence of short preambles), if it is the destination, or can decide to go back to sleep if it is not the destination. $\mathrm{X}-\mathrm{MAC}$ also enables the intended receiver to acknowledge the short preamble, which notifies the sender to initiate the transmission immediately, rather than having to wait for the completion of the whole sequence of short preambles. Thus, $\mathrm{X}$-MAC uses overhearing to reduce energy consumption and latency. X-MAC scales well with the network density, as nodes do not spend a large amount of energy while receiving unintended long preambles. X-MAC handles multiple senders in the following way. Senders refrain from sending short preambles if they detect another sender sending its preamble. After a frame exchange has been completed, senders that were waiting for the channel perform a random backoff. This backoff thus removes the synchronization of senders. Also, each sender listens to short preambles sent by other senders in the neighborhood. If a sender $s_{2}$ detects a short preamble from another sender $s_{1}$ for the receiver $r, s_{2}$ checks whether it has frames for $r$ or not. If it has, $s_{2}$ attempts to send its frames quickly after the end of the frame exchange between $s_{1}$ and $r$, with a small random backoff. The main drawbacks of X-MAC are its lack of support of QoS and its energy consumption. We plan to address these two drawbacks.

\section{PROPOSITION}

In this paper, we propose an asynchronous, sender-initiated MAC protocol for volcano monitoring in a WSN. Our protocol shares many similarities with X-MAC (which is why we used $\mathrm{X}-\mathrm{MAC}$ as the basis for comparison), but our protocol is able to provide QoS differentiation by distinguishing two types of traffic: priority traffic, and periodic traffic. Our protocol also benefits from the fact that in our volcano monitoring application, data is forwarded to a single sink, located far away from the hazardous area.

In a nutshell, our protocol uses the following mechanisms. A duty-cycle is used to parametrize the energy-consumption of the protocol in the case of periodic traffic. Priority traffic is sent using sender-initiated communications (similar as $\mathrm{X}$ MAC), which yields a low delay at the cost of high energy consumption. Periodic traffic is sent using opportunistic communications, which is achieved using low energy consumption but yields potentially large delays. Delays are reduced by taking advantage of the many-to-one paradigm: a node can send the data to any neighbor closer to the sink, rather than waiting for a specific neighbor to wake up.

In our MAC protocol, a node switches between three states: sleeping, listening and sending. (1) In the sleeping state, the node has its radio module deactivated, and saves energy. The node switches to the listening state when an urgent frame is generated by the application, or according to its duty-cycle. (2) In the listening state, the node waits to receive potential frames by listening to the channel for short preambles. When the end of a short preamble is detected, the receiver determines whether it is closer to the sink than the sender or not (using a distance metric). If it is the case, the receiver sends an early ACK after a randomized backoff, to reduce the risk of collisions among nodes closer to the sink than the sender. Upon receiving the early $\mathrm{ACK}$, the sender sends the data frame. Upon receiving this frame, the receiver sends an ACK, and starts listening for other preambles. The node switches to the sleeping state at the end of its activity duration, provided that it has no urgent frames to send. The node switches to 
the sending state when it does not detect preambles, and if it has frames to send (either urgent or periodic). (3) In the sending state, the node has data to send. It performs two clear channel assessments in order to obtain the medium access, and starts sending short preambles. Between each short preamble, the node introduces a small delay to allow the reception of early ACKs by potential receivers (including the time required for the random backoff). Upon receiving the early ACK, the sender immediately sends the frame, and waits for an ACK. If no ACK is received, the frame is retransmitted immediately (without having to wait for another early ACK). If an ACK is received, the node starts sending a new preamble for the next frames. The node switches to the sleeping state when it has no more urgent traffic to send, or at the end of its activity duration if it has only periodic traffic in queue. Note that urgent traffic is always sent before periodic traffic. Nodes use two different traffic queues for this purpose.

\section{Simulation Results}

In this section, we evaluate our MAC protocol by simulation, and we compare it with two variations of the X-MAC protocol. The first variation, called Tree X-MAC, considers that the routing protocol determines for each node a single next-hop to the sink, resulting into a tree topology. The second variation, called DAG (Directed Acyclic Graph) XMAC, considers that the routing protocol allows each node to route to any neighbor closer to the sink, resulting into a destination-oriented directed acyclic graph topology. Note that our protocol uses the same DAG topology as DAG X-MAC.

In Subsection IV-A, we describe our parameters, as well as our performance metrics. In Subsection IV-B, we focus on a scenario where only urgent traffic is generated. The main goal of this scenario is to compare our MAC protocol with X$\mathrm{MAC}$, as they share many similarities for the urgent traffic. In Subsection IV-C, we focus on a more realistic scenario where both urgent traffic and periodic traffic are generated.

\section{A. Parameter settings and performance metrics}

We developed a discrete-time simulator in $\mathrm{C}$ to implement the protocols. We considered topologies of 60 nodes randomly distributed over a $1000 m \times 1000 m$ area. The communication range is set to $100 \mathrm{~m}$. This large communication range is set to represent communications in open-space area (as vegetation is scarce on volcanoes) using a low frequency band of $433 \mathrm{MHz}$. We consider that the channel is lossless, and assume that a data frame transmission lasts for $10 \mathrm{~ms}$. This large duration could account for retransmissions in a lossy context. The sink node is chosen to be the node closest to the middle of any border of the area. On average, each node has approximately 6 neighbors. We considered the following default parameters (unless specified otherwise): the duty-cycle is set to $5 \%$, the cycle duration is set to $10 \mathrm{~s}$, the frame queue size is set to 15 frames, and 1 frame is generated every 200 s for 2 hours. Simulation results are averaged over 100 repetitions.

To evaluate our protocol, we use three performance metrics. (1) The latency is the time interval between the first transmission of a data frame by the source and its first reception by the sink. Latency only takes in account the frames that are correctly received. (2) Frame loss is defined as the ratio of the number of data frames not received by the sink over the number of data frames generated by the source nodes. The frame loss ratio takes into account the losses due to queue overflows. (3) The real duty-cycle is defined as the proportion of time when nodes are active. The real duty-cycle is a good indication of the energy consumption, as WSN nodes consume a similar amount of energy when sending frames, receiving frames or listening for frames.

\section{B. Scenario with urgent traffic}

In this scenario, each source generates an urgent frame every $200 \mathrm{~s}$ for 2 hours. 10 source nodes are chosen randomly among the nodes (sink excluded). We compare our MAC protocol with both DAG X-MAC and Tree X-MAC. We also study the effect of queue overflows in our MAC protocol by evaluating a version of our MAC protocol with unlimited frame queues. This version is described as without queue limitations (or without queue for short).

The percentage of frame loss is $0 \%$ for duty-cycles varying from $3 \%$ to $7 \%$, for all protocols. As the channel is lossless, queue overflows are the main source of losses. However, all protocols are able to handle one urgent frame per source every $200 \mathrm{~s}$, without filling the queues.

Figure 1 shows the maximum latency below which $90 \%$ of urgent frames are received by the sink, as a function of the cycle duration. When the cycle duration is large (and for a fixed duty-cycle of 5\%), nodes are inactive for larger durations. Thus, frames have to wait longer to be forwarded to the next hop. Our MAC protocol achieves lower latencies than both XMAC variations because in our protocol, nodes remain active as long as they have a frame to send. By comparing the latency between Tree X-MAC and DAG X-MAC, we can see that using a DAG significantly reduces the latency. Overall, our protocol achieves a reduction of about $10 \mathrm{~s}$ on average when compared to DAG X-MAC.

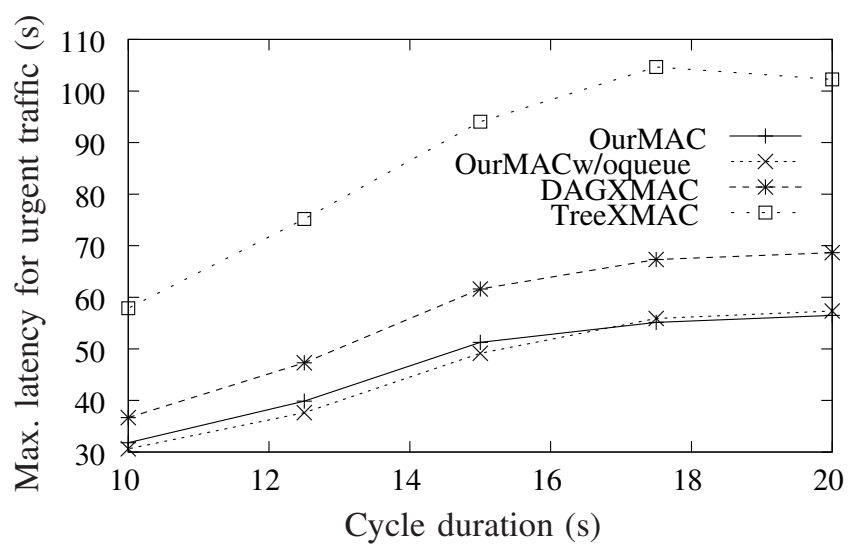

Figure 1. When nodes have urgent frames in queue, they remain active (without considering their duty-cycle). Thus, our MAC protocol achieves lower latency than both X-MAC variations.

Figure 2 shows the overall energy consumption as a function of the frame generation frequency. The frame generation period varies between $150 \mathrm{~s}$ and $250 \mathrm{~s}$ between frames. We 
notice that our MAC protocol consumes more energy than $\mathrm{X}$-MAC. Indeed, when nodes have urgent frames in queues in our MAC protocol, they remain active. In X-MAC, nodes use their duty-cycle energy-saving mechanism even when they have urgent frames in queue.

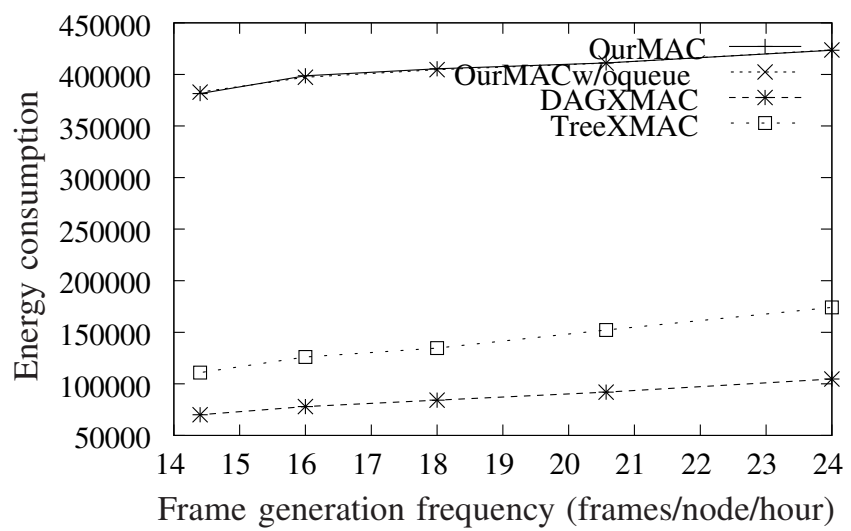

Figure 2. Energy consumption is larger for our MAC protocol than for $\mathrm{X}-\mathrm{MAC}$ as nodes are always active.

To summarize, our MAC protocol is able to transmit urgent frames faster than the X-MAC protocol, at the cost of higher energy consumption. Note that the approaches based on the DAG (that is, both DAG X-MAC and our MAC protocol) significantly reduce latency and energy consumption compared to the approach based on the tree (that is, Tree X-MAC).

\section{Scenario with mixed traffic}

In this scenario, each node generates periodic frames, and $10 \%$ of nodes are sources for the urgent traffic, with both traffic generated with the same frequency. The goal of this scenario is to show how our MAC protocol handles QoS differentiation. The queue size of both X-MAC variation is set to 15 frames. Our MAC protocol uses two queues: the periodic traffic queue size is set to 10 frames, and the urgent traffic queue size is set to 5 frames.

Figure 3 shows the maximum latency for urgent traffic as a function of the duty-cycle (used for periodic frames). We notice that the duty-cycle does not have a major impact on maximum latency. Indeed, for all protocols, frames experience a maximum latency when each sender has to wait for the receiver during a nearly full cycle. Thus, the main factors of maximum latency are cycle duration and maximum number of hops from a source to the sink, rather than duty-cycle. The variability of the Tree X-MAC results come from the variability of the length of the longest paths for each topology. It can be observed that the maximum latency with Tree XMAC is significantly larger than with all the other protocols (due to the limitations imposed by the tree routing), and that our MAC protocol is able to achieve smaller latencies for the urgent traffic, even in the presence of periodic traffic.

Figure 4 shows the percentage of urgent frame loss, as a function of the duty-cycle. We notice that the loss is negligible for all the protocols (it is about $1.4 \%$ for Tree X-MAC, and less than $0.4 \%$ for our MAC protocol). Indeed, as the channel is considered lossless, the frame loss is due to queue drops

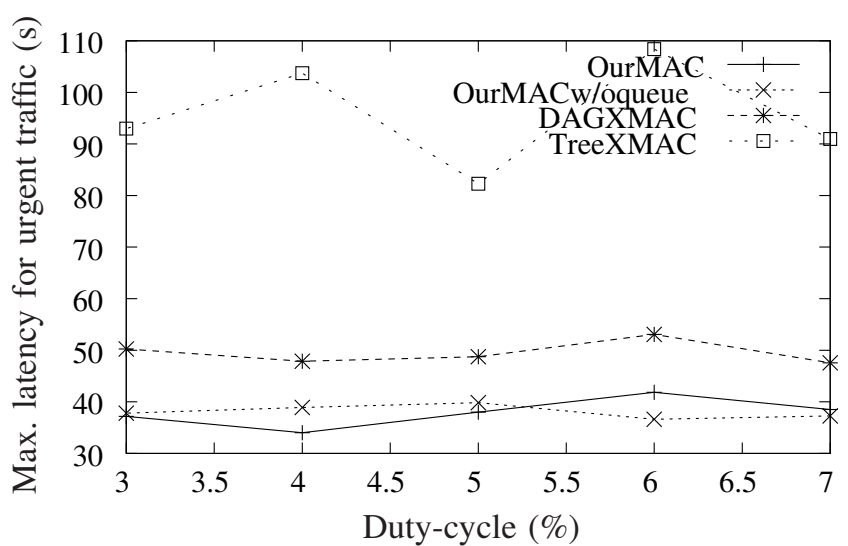

Figure 3. Maximum latency for urgent traffic, for a scenario with mixed traffic. The duty-cycle in our MAC protocol is used for the periodic traffic only.

mostly. This result show that the network load fits the network capacity, and that further latency results can be compared in a fair way (as latency only takes into account received frames).

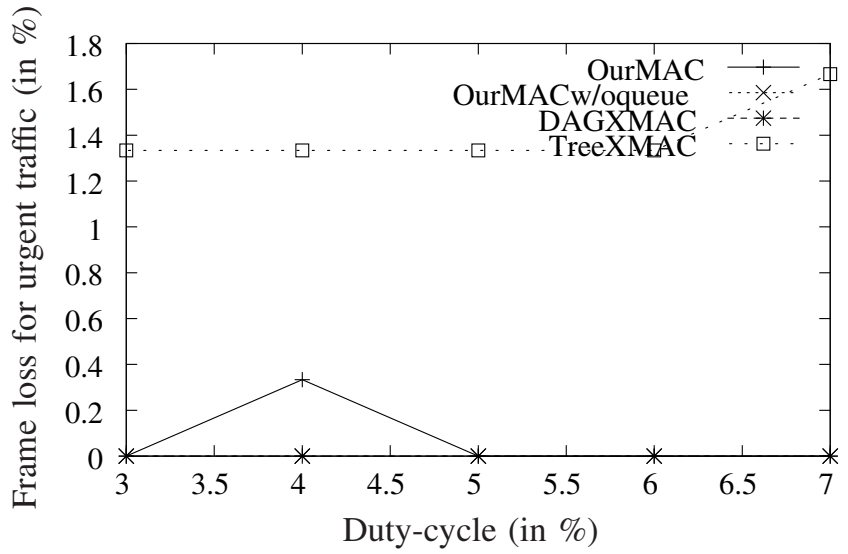

Figure 4. Frame loss is negligible for all the protocols: the network load fits the network capacity.

Figure 5 shows the percentage of frame loss for periodic traffic as a function of the duty-cycle. The percentage of frame loss is small for both X-MAC variations (it is null for DAG XMAC, and less than 5\% for Tree X-MAC). However, our MAC protocol yields between $15 \%$ and $30 \%$ frame losses. This is due to the small size of the periodic frame queue (which is only 10 in our MAC protocol, instead of 15 for X-MAC). Our MAC protocol allows some periodic frames to be lost in order to save energy and delay. Indeed, we assume that periodic frames have a less priority than urgent frames. It can be noticed that when the queue size limitation is removed from our MAC protocol, there are no more packet losses.

Figure 6 shows the average latency for periodic traffic as a function of the duty-cycle. Both X-MAC variations show that the duty-cycle has a small influence on average latency, as mentioned earlier. In our protocol, it can be noticed that the latency decreases when the duty-cycle increases. Indeed, when the duty-cycle is large, the probability that nodes communicate with each other increases, and thus frames wait less in queues. When there is no queue limitation, the latency is higher than when there is a queue limitation, because when there is a queue 


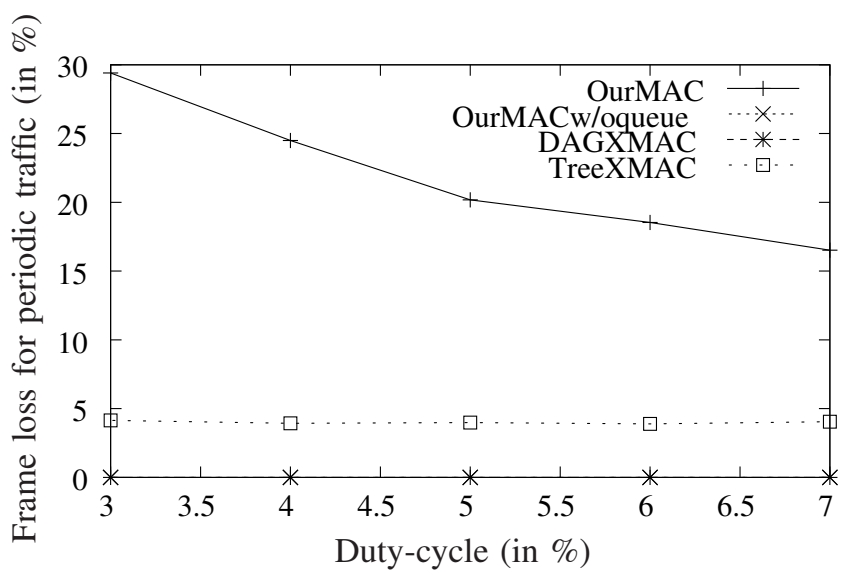

Figure 5. Frame loss for periodic traffic is important for our MAC protocol due to the priority given to urgent traffic.

overflow, frames that are dropped tend to be frames having waited for a long time in queue, which artificially reduces the latency. X-MAC shows better performance in terms of latency than our MAC protocol. This is due to the fact that our protocol considers that periodic frames have no delay constraints, and thus does not attempt to transmit them quickly. It can be seen that on average, our MAC protocol sends periodic frames about four times slower than DAG X-MAC.

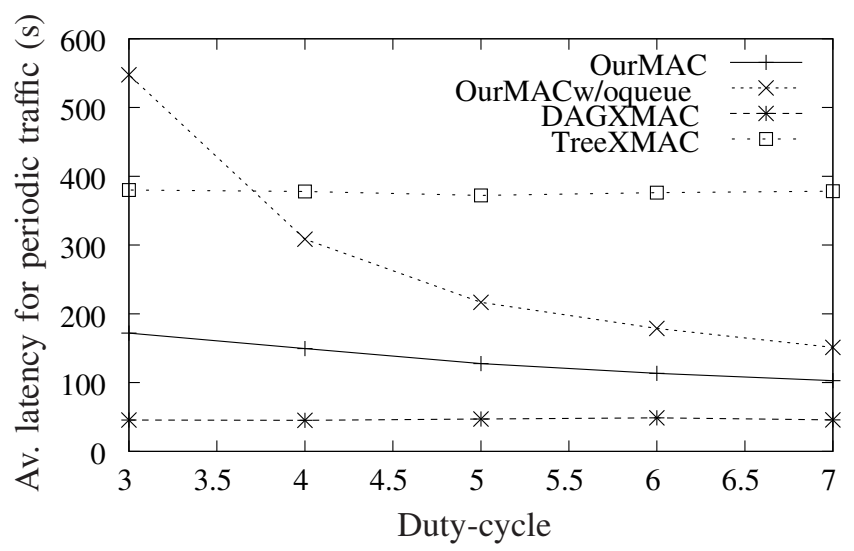

Figure 6. Periodic traffic is not a priority. Therefore, it waits more time before it is treated especially when urgent frames are generated.

Figure 7 shows the real duty-cycle (which measures the consumed energy) as a function of the parametrized dutycycle. Recall that urgent frames increase the real duty-cycle compared to the parametrized duty-cycle. It can be seen that our MAC protocol provides a good trade-off in terms of energy consumption, especially when the parametrized duty-cycle is low. The duty-cycle of X-MAC is constant (it depends on the activity duration and on the cycle duration).

\section{CONClusion}

Volcano monitoring applications based on WSNs generally produce two types of traffic: periodic traffic and urgent traffic. When nodes produce periodic traffic, the main goal is to increase the network lifetime by reducing the energy consumption, and delay is usually not an issue. However, when nodes produce urgent traffic, the main goal is to forward this traffic quickly, without focusing on energy savings. In this paper,

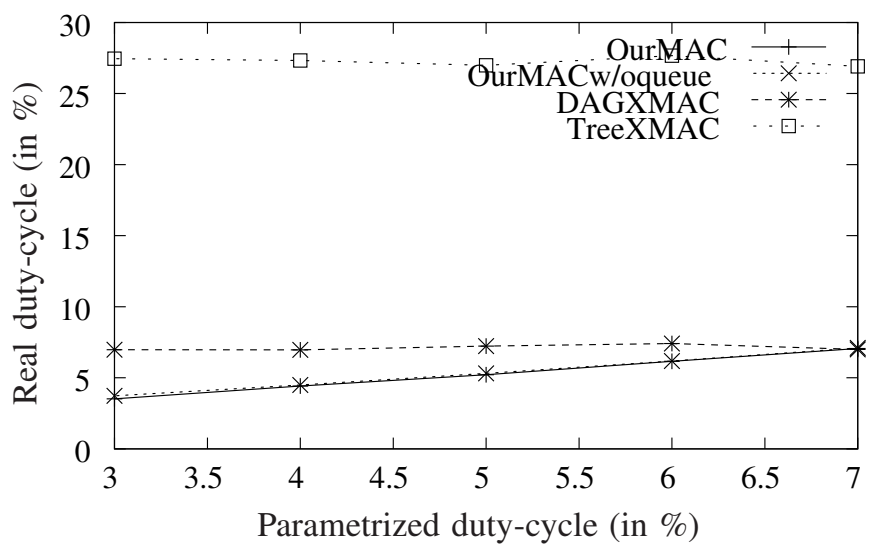

Figure 7. For X-MAC, both real and parametrized duty-cycles are similar.

we have proposed a MAC protocol that can achieve traffic differentiation. Our MAC protocol achieves a good trade-off between energy savings for the periodic traffic, and small latency for the urgent traffic. We show by simulation that our MAC protocol fills the gap of other MAC protocols of the literature, such as X-MAC.

Acknowledgment: This work has been partially funded by the Clervolc laboratory of excellence.

\section{REFERENCES}

[1] G. Barrenetxea, F. Ingelrest, G. Schaefer, and M. Vetterli, "Wireless sensor networks for environmental monitoring: The sensorscope experience," in IEEE International Zurich Seminar on Communications (IZS), 2008.

[2] D. Chen, Z. Liu, L. Wang, M. Dou, J. Chen, and H. Li, "Natural disaster monitoring with wireless sensor networks: A case study of dataintensive applications upon low-cost scalable systems," Mobile Network Applications, vol. 18, pp. 651-663, 2013.

[3] J. Polastre, J. Hill, and D. Culler, "Versatile low power media access for wireless sensor networks," in SenSys (ACM Conference on Embedded Networked Sensor Systems), 2004, pp. 95-107.

[4] I. F. Akyildiz, W. Su, Y. Sankarasubramaniam, and E. Cayirci, "Wireless sensor networks: A survey," Computer Networks, vol. 38, no. 4, pp. 393 422, 2002.

[5] P. Huang, L. Xiao, S. Soltani, W. Mutka Matt, and N. Xi, "The evolution of MAC protocols in wireless sensor networks: A survey," Communications Surveys Tutorials, IEEE, vol. 15, no. 1, pp. 101-120, 2013.

[6] H. Tang and J. Cao, "SR-MAC: A low latency MAC protocol for multipacket transmissions in wireless sensor networks," Journal of Computer Science and Technology, vol. 28, no. 2, pp. 329-342, 2013.

[7] W. Ye, J. Heidemann, and D. Estrin, "An energy-efficient MAC protocol for wireless sensor networks," in INFOCOM Twenty-First Annual Joint Conference of the IEEE Computer and Communications Societies. Proceedings. IEEE, vol. 3, 2002, pp. 1567-1576.

[8] Y. Sun and S. Du, "DWMAC: A low latency, energy efficient demandwakeup MAC protocol for wireless sensor networks," in ACM MOBIHOC, 2008.

[9] IEEE 802.15, "Part 15.4: low-rate wireless personal area networks (LRWPANs)," IEEE, Standard for local and metropolitan area networks IEEE Std 802.15.4-2011, 2011.

[10] H. Tang, C. Sun, Y. Liu, and B. Fan, "Low-latency ashynchronous dutycycle MAC protocol for burst traffic in wireless sensor networks," in IEEE IWCMC, 2013.

[11] A. El-Hoiydi and J.-D. Decotignie, "WiseMAC: An ultra low power MAC protocol for the downlink of infrastructure wireless sensor networks," in Proceedings of the Ninth IEEE International Symposium on Computers and Communications (IEEE ISCC), 2004, pp. 244-251.

[12] M. Buettner, G. V. Yee, E. Anderson, and R. Han, "X-MAC: A short preamble MAC protocol for duty-cycled wireless sensor networks," in Proceedings of the 4th International Conference on Embedded Networked Sensor Systems, 2006, pp. 307-320. 